\title{
A Wireless, Passive, Magnetically-soft Harmonic Sensor for Monitoring Sodium Hypochlorite Concentrations in Water
}

\author{
Keat G. Ong ${ }^{1}$, Maggie Paulose ${ }^{1}$ and Craig A. Grimes ${ }^{2, *}$ \\ ${ }^{1}$ SenTech Corporation, 200 Innovation Blvd. Suite 236, State College, PA 16803 \\ ${ }^{2}$ Department of Electrical Engineering and Materials Research Institute 217 Materials Research Lab, \\ The Pennsylvania State University, University Park, PA 16802
}

* Author to whom correspondence should be addressed. Email: cgrimes@engr.psu.edu

Received: 20 November 2002 Accepted: 5 December 2002 Published: 25 January 2003

\begin{abstract}
A wireless, passive, remote-query sensor for monitoring sodium hypochlorite (bleach) solutions is reported. The sensor is comprised of a magnetically-soft ferromagnetic ribbon, coated with a layer of polyurethane and alumina, having a large and nonlinear permeability that supports higher-order harmonics in response to a time varying magnetic field. The hypochlorite ions induce swelling in the coating, with the resultant stress altering the harmonic signature of the sensor from which the sodium hypochlorite concentration can be determined. The wireless, passive nature of the sensor platform enables long-term monitoring of bleach concentrations in the environment. The sensor platform can be extended to other chemical analytes of interest as desired.
\end{abstract}

Keywords: Wireless sensor, passive, bleach, chlorine, higher-order harmonics.

\section{Introduction}

Bleach, commonly a $5.75 \mathrm{vol} \%$ sodium hypochlorite, $\mathrm{NaOCl}$, solution, is an intensively used industrial chemical widely used for cleaning due to its properties as a disinfectant. While bleach is an excellent cleaning solution, at high concentrations it can readily damage drainage pipes. Hence the ability to remotely monitor sodium hypochlorite concentrations from within sealed pipes is of great interest for ensuring environmental integrity; if excessive quantities of bleach are detected appropriate steps can be taken to neutralize the chemical.

In this paper we present a wireless, passive sensor for in situ monitoring of sodium hypochlorite in water. The sensor is made of a high permeability, magnetically soft, ribbon-like amorphous 
ferromagnetic alloy that supports higher-order harmonics [1-4] in response to a magnetic ac field. In this work, two types of ferromagnetic alloys $\mathrm{Fe}_{40} \mathrm{Ni}_{38} \mathrm{Mo}_{4} \mathrm{~B}_{18}$ (Metglas 2826MB) and $\mathrm{Co}_{65} \mathrm{Fe}_{4} \mathrm{Ni}_{2} \mathrm{Si}_{15} \mathrm{~B}_{14}$ (Metglas 2714a), from Honeywell Co. [5], are investigated. The sensors are first coated with a layer of polyurethane to prevent rusting of the iron-rich material, followed by a layer of alumina. The coating swells in response to changing bleach concentration, varying the stress and hence the magnetic permeability of the sensor and consequently the relative amplitudes of the higherorder harmonics generated by the sensor in response to an interrogation signal. Between these two materials, Metglas 2826MB is highly sensitive to stress (and hence bleach concentration) due to its high magnetostriction of $12 \mathrm{ppm}$. Metglas 2714a, on the other hand, has a magnetostriction of less than 1 and is insensitive to stress. The magnetic properties of both Metglas are listed in Table 1.

Table 1. Magnetic properties of Metglas 2826MB and Metglas 2714a.

\begin{tabular}{|c|c|c|c|c|c|}
\hline Material & $\boldsymbol{H}_{\boldsymbol{k}}(\mathbf{A} / \mathbf{m})$ & $\boldsymbol{H}_{\boldsymbol{c}}(\mathbf{A} / \mathbf{m})$ & $\boldsymbol{B}_{\boldsymbol{s}}(\mathbf{T})$ & $\boldsymbol{\mu}(\mathbf{d c})$ & $\boldsymbol{\lambda}_{\boldsymbol{s}}(\mathbf{p p m})$ \\
\hline $2826 \mathrm{MB}$ & 150 & 4 & 0.88 & 50,000 & 12 \\
\hline $2714 \mathrm{a}$ & 60 & 2 & 0.57 & 80,000 & -1 to 0.1 \\
\hline \multicolumn{5}{c}{$\begin{array}{c}\text { Spectrum } \\
\text { Analyzer }\end{array}$} & $\begin{array}{l}\text { Function Generator } \\
\text { \& DC Power Supply }\end{array}$
\end{tabular}

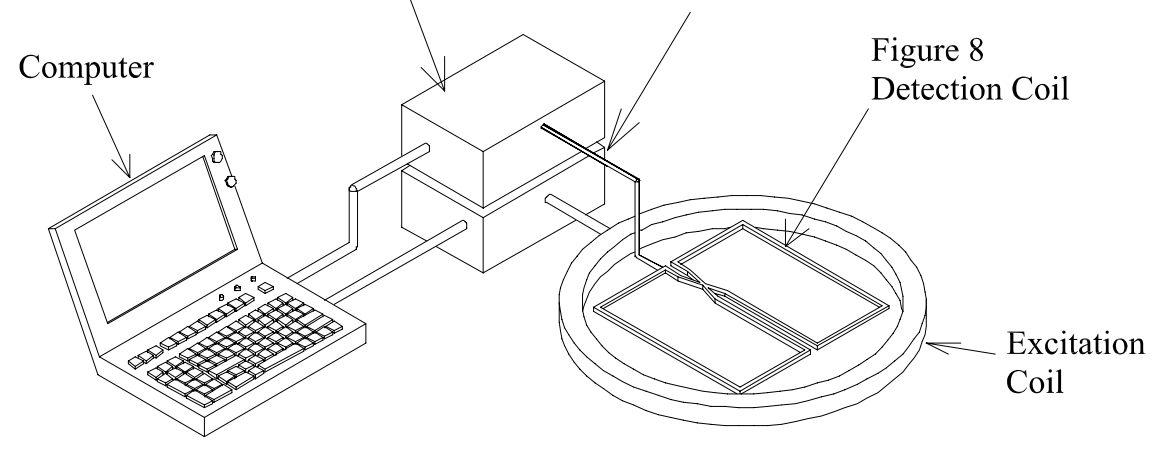

Sensor

Figure 1. Experimental set up for monitoring the harmonic response of a magnetically soft sensor.

The sensor is placed in the environment of interest as shown in Fig. 1. The sensor is excited by a single interrogation coil, and the higher-order harmonic response is detected by a figure- 8 sensing coil coplanar with the interrogation coil. Since there is no coupling between the measured higher-order harmonic signals and the excitation signal the background noise level is relatively small enabling a greater detection range. Since the response is based upon the magnetic properties of the material, sensor length does not affect the response except total signal amplitude, where a physically larger sensor provides more signal than a physically smaller sensor. The only size restriction is when the dimensions of the sensor are reduced to a point that the demagnetizing field (associated with all magnetic materials) reduces the permeability of the material. The sensors used in this work, $55 \mathrm{~mm} \times 6.35 \mathrm{~mm} \times 30 \mu \mathrm{m}$, have a material cost of less than $\$ 0.005$ making disposable use practical. 
The sensors were excited using a coil (36-turn, 14 gauge wire, $30 \mathrm{~cm}$ radius) connected to a Wavetek 20 function generator to generate a $500 \mathrm{~A} / \mathrm{m} 200 \mathrm{~Hz}$ magnetic field, in series with a Kepco dc power supply used to provide a dc biasing field of controllable amplitude. A figure- 8 sensing coil (400-turn, 40 gauge wire, $18 \mathrm{~cm} \times 18 \mathrm{~cm}$ total area) co-planar with the interrogation coil was used to monitor the response of the sensor, with the harmonic amplitudes captured using a HP4293B spectrum analyzer. A computer was used to automate the experiment.

The sensor was fabricated by dip coating an as-cast Metglas $2826 \mathrm{MB}$ ribbon with polyurethane and then drying at $40^{\circ} \mathrm{C}$ for two hours. The dip coating process was repeated three times for a total polyurethane layer thickness of $200 \mu \mathrm{m}$. An alumina coating was then applied, via three separate dip coatings, having a total thickness of approximately $50 \mu \mathrm{m}$. Finally the sensor was baked in an oven at $100^{\circ} \mathrm{C}$ for 3 hours.

\section{Theory}

The amplitude of the higher order harmonics of a ribbon-shaped soft magnetic material is dependent upon the magnitude of the biasing field. From our previous work [6-9], the amplitude of the $n$-th mode higher-order harmonics, $A_{n}$, as a function of biasing field $H_{d c}$ is given by:

$$
A_{n}=\frac{L B_{s} \omega}{\omega^{2}} \cdot\left(e^{\frac{j n \pi H d x}{h_{\text {ti }}}} \cos (\pi n)-k \mu \sin \left(\frac{1}{k \mu}\right)\right)
$$

where $\omega$ is the radian frequency of the excitation field, $h_{a c}$ is the amplitude of the ac excitation field, $B_{s}$ is the saturation induction of the magnetic material, $\mu$ is the permeability of the material, $L$ is the coupling between the magnetic ribbon and the detection coil, and $k=h_{a c} / \pi n B_{s}$. Eq. (1) shows the harmonic amplitudes, regardless of the order number and the amplitude of biasing field, increase with permeability.

When the magnetic ribbon is exposed to a longitudinal stress $\sigma$, such as the stress induced by the swelling of the bleach-sensing layer, its anisotropy field changes [11-13]:

$$
H_{k}^{\sigma}=H_{k}-\frac{3 \lambda_{s} \sigma}{M_{s}}
$$

where $\lambda_{s}$ is the saturation magnetostriction, $H_{k}$ is the anisotropy field at zero stress, $H_{k}^{\sigma}$ is the anisotropy field at stress $\sigma$, and $M_{s}$ is the saturation magnetization of the material $\left(M_{s} \approx B_{s}\right.$ for our sensor since it has a high $\mu$ ). The permeability of the sensor at stress $\sigma$ is determined using the approximation $\mu \approx B_{s} / H_{k}$ as:

$$
\mu=\frac{B_{s}^{2}}{B_{s} H_{k}-3 \lambda_{s} \sigma}
$$

Eq. (3) shows the permeability of the magnetically soft material increases with increasing longitudinal stress $\sigma$. The measured harmonic amplitudes at biasing field $H_{d c}$ and applied stress $\sigma$ are:

$$
A_{n}=\frac{L B_{s} \omega}{\pi^{2}}\left(e^{j n \pi H_{d x} / h_{\alpha x}} \cos (\pi n)-\frac{k B_{s}{ }^{2}}{B_{s} H_{k}-3 \lambda_{s} \sigma} \sin \left(\frac{B_{s} H_{k}-3 \lambda_{s} \sigma}{k B_{s}^{2}}\right)\right)
$$


From Eq. (4) it is clear the amplitudes of the higher-order harmonics increase with increasing stress. As a result, the higher-order harmonics of the sensor is expected to increase with sodium hypochlorite concentration since the absorption of the hypochlorite ions causes the sensing layer to swell, which stresses the magnetic ribbon.

Our sensor is encapsulated within a thin coating of polyurethane and alumina as shown in Fig. 2. When the sensor is immersed into a sodium hypochlorite solution, the coating absorbs the sodium hypochlorite ions and swells introducing stress as illustrated in Fig. 2. Since the sensor has a high aspect ratio the longitudinal stress will be much larger than the transverse stress, with the stress changing the magnetic permeability of the sensor and in turn the relative amplitude of the different harmonics.

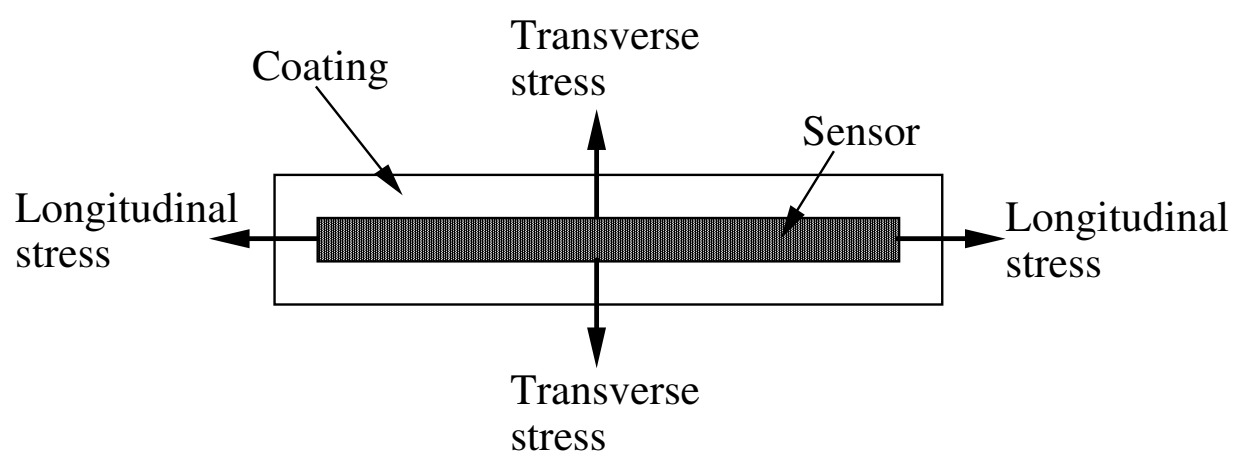

Figure 2. Schematic drawing illustrating stresses induced by coating swelling.

\section{Results and Discussions}

Fig. 3 plots the amplitudes of the $2^{\text {nd }}$ and $3^{\text {rd }}$ harmonics of a typical sensor as a function of dc biasing field. To determine the maximum amplitudes of both even and odd harmonics, a dc sweep is first performed to capture the harmonic response as a function of dc biasing fields. A peak-finding routine is then used to determine the maximum amplitude for each harmonic.

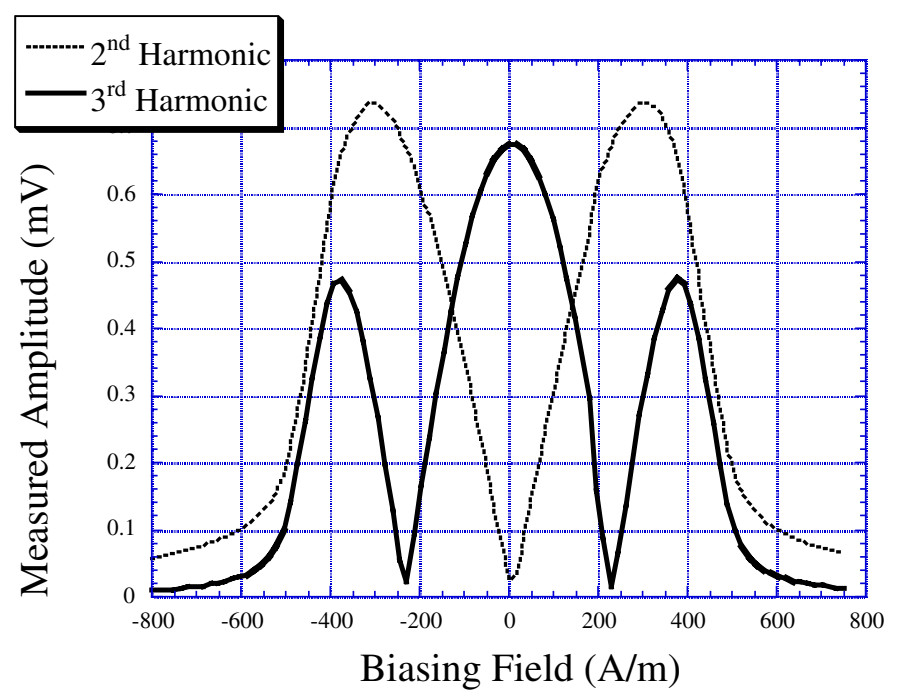

Figure 3. The measured amplitude of the 2 nd and $3 \mathrm{rd}$ harmonics versus amplitude of the dc magnetic biasing field. 
The $2826 \mathrm{MB}$ sensor was tested by immersion in a $5.75 \%$ sodium hypochlorite (commercially available bleach [14]) and deionized water. Before each experiment started the sensor was presoaked in water for 2 hours to eliminate any amplitude variations caused by the absorption of water molecules, and the associated stress, by the coating. Fig. 4 plots the variation in the $2^{\text {nd }}$ and $3^{\text {rd }}$ harmonic amplitudes when a dry sensor was immersed in deionized water. As shown in the plot, the maximum amplitudes of the higher-order harmonics increased and then saturated at constant values after 2 hours.

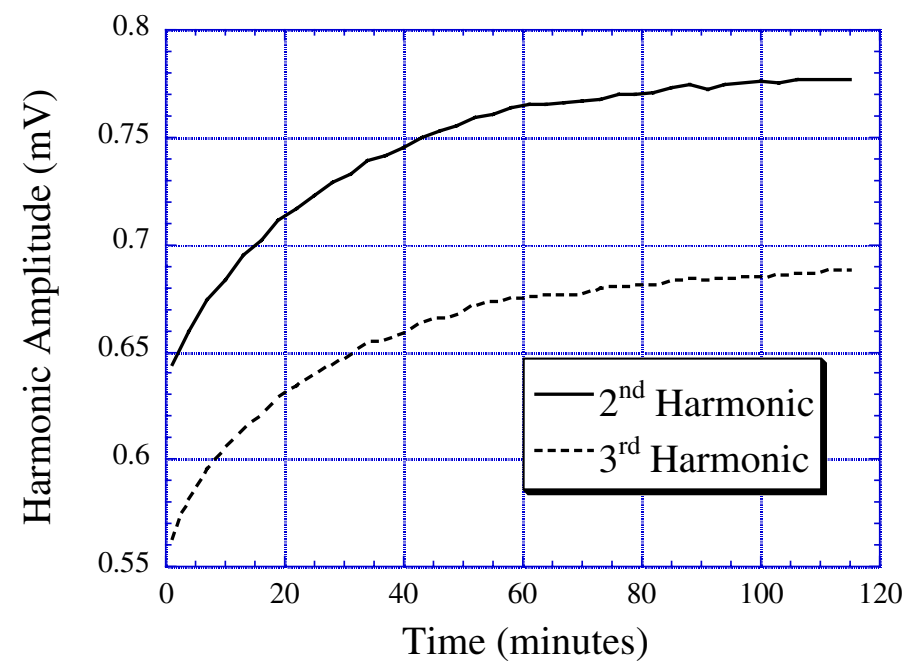

Figure 4. Measured variation in the $2^{\text {nd }}$ and $3^{\text {rd }}$ harmonic amplitudes when a dry sensor was immersed in deionized water.

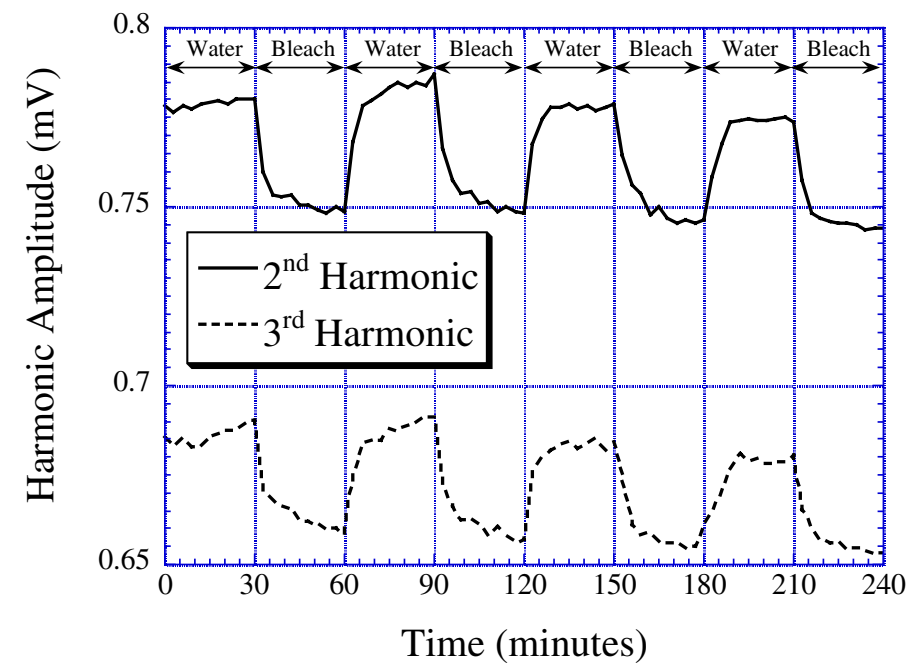

Figure 5. The changes in the $2^{\text {nd }}$ and $3^{\text {rd }}$ harmonic amplitudes when a sensor was cycled between deionized water and $5.75 \%$ sodium hypochlorite.

Fig. 5 plots the amplitudes of the $2^{\text {nd }}$ and $3^{\text {rd }}$ harmonics when the $2826 \mathrm{MB}$ sensor was cycled between deionized water and $5.75 \%$ sodium hypochlorite solution after the sensor was presoaked in water for 2 hours. The measured harmonic amplitudes decrease with each bleach/water cycle, indicating a decrease in coating swelling. The variation in the measured $2^{\text {nd }}$ and $3^{\text {rd }}$ harmonic 
amplitudes between the two test liquids were, respectively, $0.03 \mathrm{mV}$ and $0.027 \mathrm{mV}$, or about $3.7 \%$ of the measured signal amplitudes. Fig. 6 shows the sensor was able to measure the concentration of hypochlorite solution diluted in water at varying concentrations; the sensors were immersed in the test solution for thirty minutes and then measured. The sensor responded linearly to bleach concentration, with a high/low hysteresis of less than $10 \%$.

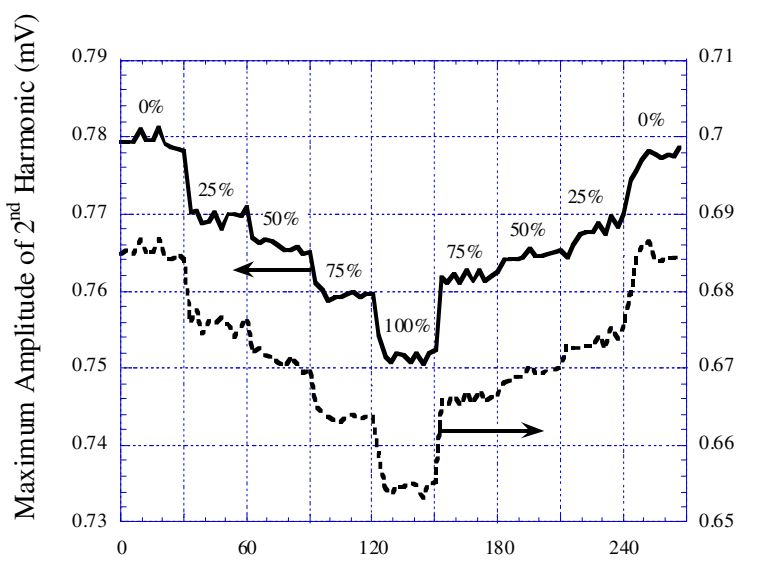

a)

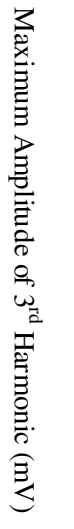

b)

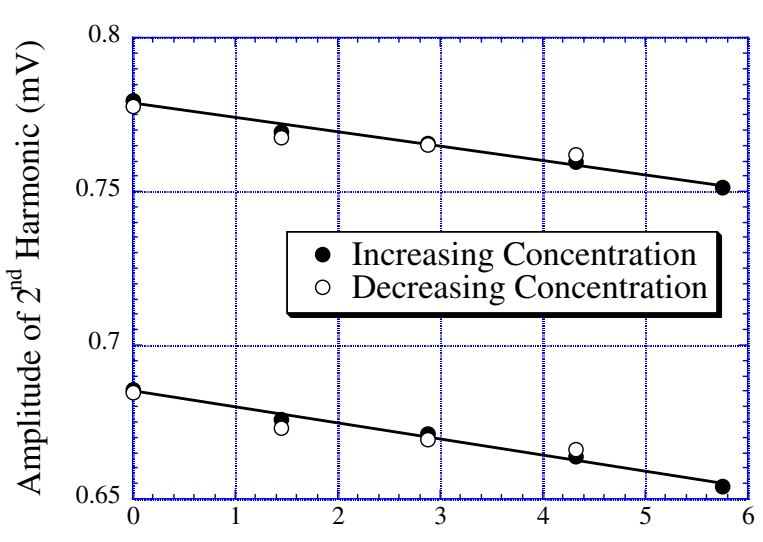

Sodium Hypochloride Concentration (\%vol)

Figure 6. (a) The harmonic amplitudes of the sensor when it was exposed to different sodium hypochlorite concentrations. The numbers in the figure are the concentrations of sodium hypochlorite in water. (b) The sensor shows a linear response towards bleach, and the hysteresis between increasing and decreasing concentration is less than $10 \%$.

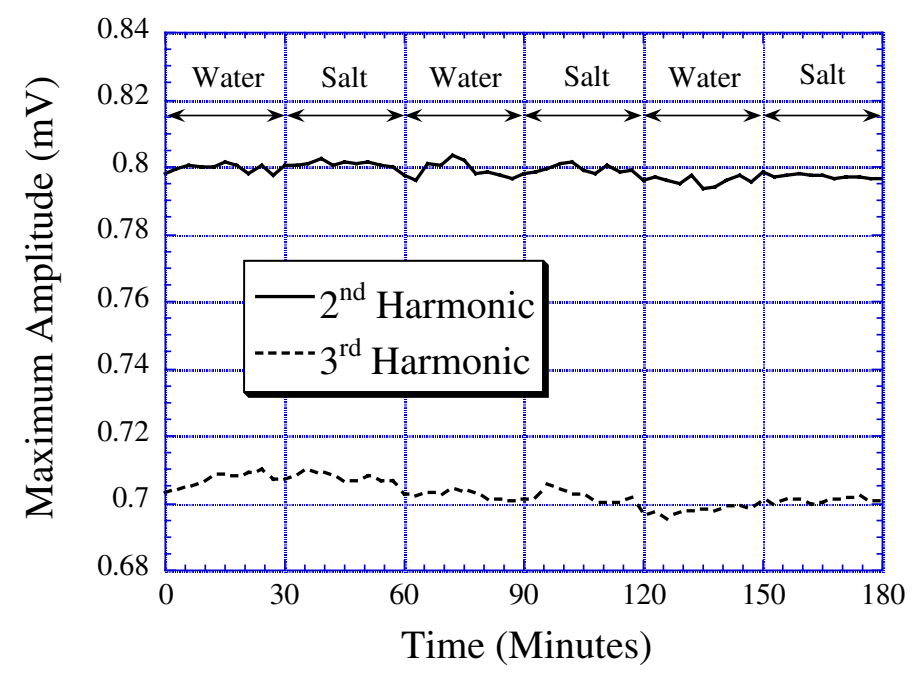

Figure 7. The $2^{\text {nd }}$ and $3^{\text {rd }}$ harmonic amplitudes stayed constant when the sensor was cycled between water and water containing $5.2 \mathrm{~g} / \mathrm{l}$ sodium chloride $(\mathrm{NaCl})$.

Fig. 7 shows the response of the $2826 \mathrm{MB}$ sensor when cycled between deionized water and water containing salt (sodium chloride $5.2 \mathrm{~g} / \mathrm{l}$ ). As shown in the plot, the sensor is insensitive to chloride ions. 
Eq. (4) indicates that the stress-response of the sensor is proportional to the magnetostriction of the sensor material $\lambda_{s}$. Hence, the sensitivity of the sensor should increase with $\lambda_{s}$ of the sensor material. Fig. 8 plots the response when a sensor made of Metglas 2714a $\left(\mathrm{Co}_{65} \mathrm{Fe}_{4} \mathrm{Ni}_{2} \mathrm{Si}_{15} \mathrm{~B}_{14}\right.$, see Table 1 for its magnetic properties) was cycled between deionized water and the sodium hypochlorite solution. Since Metglas 2714a has a magnetostriction of less than $1 \mathrm{ppm}$, in contrast to Metglas 2826MB with magnetostriction of $12 \mathrm{ppm}$, it is insensitive to chlorine as indicated in the plot.

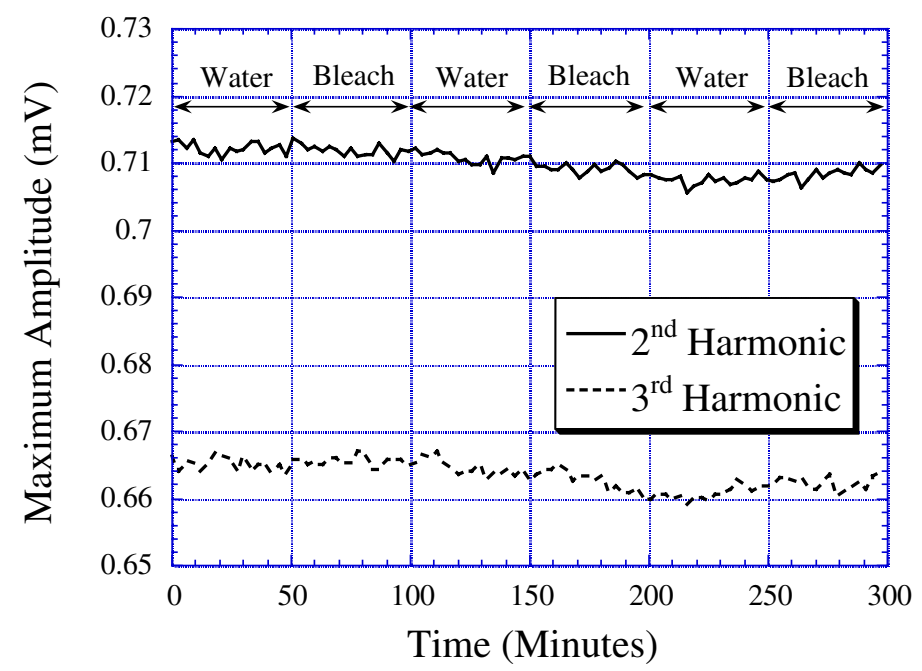

Figure 8. The $2^{\text {nd }}$ and $3^{\text {rd }}$ harmonic amplitudes remained unchanged when a sensor made of Metglas 2714a was cycled between deionized water and $5.75 \%$ sodium hypochlorite. This sensor is insensitive to bleach since Metglas 2714a has a low magnetostriction of less than $1 \mathrm{ppm}$.

\section{Conclusions}

A wireless sensor useful for monitoring sodium hypochlorite concentrations has been presented. The sensor is a magnetically soft ribbon that supports higher-order harmonics the characteristics of which change with stress. The sensor is coated with a layer of polyurethane and alumina, the bleach dependent swelling of which induces stress in the sensor changing the amplitudes of the higher order harmonics. The sensor is interrogated through an excitation coil that generates a fixed-frequency ac field mixed with a dc field of varying amplitude. The harmonic response of the sensor as a function of dc biasing field is captured with a figure- 8 detection coil, and the peak of each harmonic is determined for tracking bleach concentration. A theoretical model is presented to explain how the absorption of hypochlorite ions by the alumina coating creates stresses on the sensor affecting the higher-order harmonic response. Experimental results show the harmonic sensor is sensitive to sodium hypochlorite, a corrosive chemical, but does not respond to salt water containing sodium chloride. We have also shown a magnetically soft ribbon with large magnetostriction is needed for high sensitivity.

The sensor is remotely detected, so it can operate inside a confined place, such as a PVC or concrete sewer pipe, without any electrical connections. The sensor is passive; hence there are no 
battery lifetime issues. It should be noted that although the sensor can be operated in the vicinity of magnetic objects, its accuracy will be reduced due to interference from the stray magnetic fields.

\section{Acknowledgements}

Support of this work by NASA through grant NAG-1-01036, and NSF through grants ECS-0196494 and ECS-9875104 is gratefully acknowledged.

\section{References}

1. Humphrey, F.B. Article surveillance magnetic marker having a hysteresis loop with large Barkhausen discontinuities. US Patent 4,660,025, 1987.

2. Montean, S. Magnetic asymmetric antipilferage marker. US Patent 4,075,618, 1978.

3. Fletcher, R.R.; Gershenfeld, N.A. Remote interrogated temperature sensors based on magnetic materials. IEEE Trans. Magn. 2000, 36(5), 2794-2795.

4. Bakeman, P.E.; Armstrong, A.L. Pilferage detection systems. US Patent 3,983,552, 1976.

5. Honeywell Corporation, 101 Columbia Road, Morristown, NJ 07962 USA. http://www.honeywell.com.

6. Kim, C.G.; Kim, H.C.; Ahn, S.J.; Cha, S.Y.; Chang, S.K. Magnetizing angle dependence of harmonics of magnetic induction and magnetostriction in electrical steel. J. Magn. Magn. Mater. 2000, 215-216, 159-161.

7. Ong, K.G.; Grimes, D.M.; Grimes. C.A. Higher-order harmonic of a magnetically soft sensor: Application to remote-query temperature monitoring. Appl. Phys. Lett. 2002, 80(20), 3856-3858.

8. Sablik, M.J.; Burkhardt, G.L.; Kwun, H.; Jiles, D.C. A model for the effect of stress on the lowfrequency harmonic content of the magnetic induction in ferromagnetic materials. J. Appl. Phys. 1988, 63(8), 3930-3932.

9. Ong, K.G.; Grimes, C.A. Tracking the harmonic response of magnetically-soft sensors for wireless temperature, stress, and corrosive monitoring. Sensors and Actuators A 2002 3487, 1-13.

10. Phillips, C.L.; Parr, J.M. Signals, Systems, and Transforms. Prentice Hall, NJ, USA, 1995.

11. O'Handley, R.C. Modern Magnetic Materials: Principles and Applications. John Wiley \& Sons Inc, New York, 2000, pp. 242-243.

12. Livingston, J.D. Magnetomechanical properties of amorphous metals. Phys. Stat. Sol. A 1982, 70, 591-596.

13. de Lacheisserie, T. Magnetoelastic properties of amorphous alloys. J Magn. Magn. Mater. 1982, 25, 251-270.

14. Home brand ultra-bleach, marketed by WalMart ${ }^{\circledR}$ Stores Inc. Bentonville, AR 72716.

Sample Availability: Available from the authors.

(C) 2003 by MDPI (http://www.mdpi.net). Reproduction is permitted for noncommercial purposes. 